\title{
Frauen in der beruflichen Aus- und Weiterbildung. Konsequenzen für die Geschlechterfrage in der Berufsbildung
}

\section{Silvia Grossenbacher}

Der Artikel beleuchtet Probleme und Diskriminierungen, von denen viele Frauen über die gesamte Bildungs- und Berufslaufbahn hinweg betroffen sind. Er nimmt dabei Bezug auf die verschiedenen Phasen: Berufswahl, Grundausbildung, Weiterbildung und Wiedereinstieg. Die Autorin verweist kritisch darauf, dass Massnahmen zur Gleichstellung der Geschlechter bisher einseitig auf Information und Motivation der Frauen ausgerichtet waren. Sie zeigt ergänzend dazu Ansätze auf, die strukturelle Veränderungen anstreben.

Zur Situation von Frauen in der beruflichen Aus- und Weiterbildung wurden in den vergangenen 20 Jahren zahlreiche punktuelle Studien durchgeführt. Eine systematische Bearbeitung dieser Fragestellung fehlt jedoch, auch wenn beispielsweise im Bereich der amtlichen Statistik mit zunehmender Konsequenz darauf geachtet wird, Daten geschlechtergetrennt auszuweisen und aufzuarbeiten (z.B. Bundesamt für Statistik [BFS], 1996). Dieser Mangel hängt u.a. damit zusammen, dass in der Schweiz "gender studies» oder "women's studies» kaum institutionalisiert sind, wie eine 1998 veröffentlichte Bestandesaufnahme gezeigt hat (vgl. Burri, Fleischmann und Pagnossis-Aligisakis, 1998). Ende der 90er Jahre wurde der Forschungsstand zur Situation von Frauen in der beruflichen Ausund Weiterbildung in der Schweiz erstmals umfassend dargestellt. Der Bericht zeigt Probleme und Diskriminierungen auf, von denen viele Frauen über die gesamte Bildungs- und Berufslaufbahn hinweg betroffen sind, und er macht deutlich, dass die Kampagnen zur Lösung dieser Probleme lange Zeit einseitig auf Sensibilisierung und Motivation von Frauen zur Veränderung ihrer Situation ausgerichtet waren. Der Bericht würdigt diese Bemühungen, geht in den Empfehlungen zum Abbau von geschlechtsspezifischen Diskriminierungen aber weiter. Der Artikel fasst Kernaussagen dieses Berichtes zusammen. 


\section{Ausgangslage: Parlamentarischer Vorstoss ermöglicht Situationsanalyse}

Die wirtschaftlichen Entwicklungen im Zusammenhang mit der Globalisierung liessen auch in der Schweiz Ende der 80er Jahre einen steigenden Bedarf an hoch qualifizierten Arbeitskräften erkennen. Dieser Bedarf und der wachsende Anpassungsdruck an Europa im Bereich der Bildungssysteme und der Bildungsabschlüsse leiteten entscheidende Umbauten im schweizerischen Berufsbildungssystem ein. Die bereits seit den 70er Jahren bestehenden Berufsmittelschulen wurden aufgewertet und führen neu zur Berufsmatur. Die Höheren Fachschulen wurden umgewandelt in Fachhochschulen, die einen um Forschung, Dienstleistung und Weiterbildung erweiterten Leistungsauftrag erhielten. Dieser Prozess wurde zunächst für die in Bundeskompetenz stehenden ehemaligen Höheren Fachschulen (Technik, Wirtschaft und Gestaltung) vollzogen und dann auf die unter kantonaler Hoheit stehenden ehemaligen Höheren Fachschulen (in den Bereichen Gesundheit, Soziales, Musik, Kunst, Pädagogik) ausgedehnt.

Im Zusammenhang mit diesen Umbauten reichte die Solothurner CVP-Nationalrätin Ruth Grossenbacher im Juni 1993 eine Motion zur beruflichen Ausund Weiterbildung von Frauen ein. Die Motionärin forderte den Bundesrat auf, mit geeigneten Massnahmen junge Frauen von der Wichtigkeit einer guten Ausund Weiterbildung zu überzeugen, den Anteil der Frauen an Berufsmittelschulen und den Frauenanteil an den technischen Fachhochschulen zu erhöhen und die spezifischen Weiterbildungsmöglichkeiten nach einer zweijährigen Berufslehre zu verbessern. Die Motion wurde in ein Postulat umgewandelt und überwiesen. Danach kam eine Arbeitsgruppe zum Einsatz, bestehend aus Vertreterinnen und Vertretern des damaligen Bundesamtes für Industrie, Gewerbe und Arbeit BIGA (heute: Bundesamt für Berufsbildung und Technologie BBT), des Eidgenössischen Gleichstellungsbüros und des Bundesamtes für Statistik (BFS). Diese Arbeitsgruppe erarbeitete zusammen mit zwei Expertinnen (Veronika Baumgartner, Organisationsentwicklerin, und Silvia Grossenbacher, wissenschaftliche Mitarbeiterin der Schweizerischen Koordinationsstelle für Bildungsforschung) und Fachfrauen aus der Praxis einen Bericht zur «Beruflichen Ausund Weiterbildung von Frauen».

Der Anlass gab Gelegenheit, den Forschungsstand zum Thema in der Schweiz aufzuarbeiten, die bestehenden Fördermassnahmen und Motivationskampagnen zu erfassen und die Lücken in diesem Bereich aufzuzeigen. Diese Situationsanalyse wurde ergänzt um zahlreiche Empfehlungen und Umsetzungsvorschläge für die Praxis. Nach einiger Verzögerung und einer Aktualisierung der Daten durch Irène Meier (Sozialwissenschafterin) wurde der Bericht «Berufliche Aus- und Weiterbildung von Frauen» vom Bundesamt für Berufsbildung und Technologie im Jahre 1998 veröffentlicht (Bundesamt für Berufsbildung und Technologie, 1998). Einen ersten Niederschlag fand er aber bereits im «Bericht über die Berufsbildung», den der Bundesrat im September 1996 vorgelegt 
hat. Dieses Dokument skizziert Perspektiven des Bundes im Bereich Berufsbildung und die Formulierung einer der vorgeschlagenen Massnahmen lässt sich so interpretieren, dass die Landesregierung Frauenförderung als Querschnittaufgabe in der Berufsbildung verstanden haben will. Die Formulierung lautet: «Das BIGA wird bei der Erfüllung seiner Rechtsetzungsaufgaben für die Berufsbildung dem Aspekt der Frauenförderung besondere Beachtung schenken. Insbesondere soll dies auch bei der Umsetzung der anderen im vorliegenden Bericht dargelegten Massnahmen geschehen» (Schweizerischer Bundesrat, 1996, S. 7).

Diesem Prinzip kam das genannte Bundesamt in der Folge sowohl bei der Ausgestaltung des sogenannten Lehrstellenbeschlusses wie auch bei der Vorlage für ein revidiertes Berufsbildungsgesetz nach. Der Lehrstellenbeschluss (Bundesbeschluss vom 30. April 1997 über Massnahmen zur Verbesserung des Lehrstellenangebotes für die Ausbildungsjahre 1997, 1998 und 1999) ist ein Teil des Investitionsprogrammes des Bundes zur Belebung der Wirtschaft und zielt darauf $\mathrm{ab}$, die Ausbildungsbereitschaft der Betriebe, die in den Krisenjahren gesunken ist, zu erhöhen, und gleichzeitig das Interesse junger Menschen für die Berufsausbildung zu steigern. Prioritär sind dabei das Lehrstellenmarketing sowie Motivationskampagnen, insbesondere hinsichtlich Lehrstellen für Frauen. Der Lehrstellenbeschluss wurde nach seinem Auslaufen 1999/2000 erneuert (Bundesbeschluss vom 8. Juni 1999 über die Finanzierung der Massnahmen zur Verbesserung des Lehrstellenangebotes und zur Entwicklung der Berufsbildung, Lehrstellenbeschluss II). Anlässlich dieser Erneuerung wurde der Geschlechtergleichstellung in den Umsetzungsrichtlinien ein prioritärer Stellenwert eingeräumt. Auch im Entwurf für ein neues Berufsbildungsgesetzes, der sich zur Zeit der Abfassung dieses Artikels in Vernehmlassung befindet, spielt Gleichstellung - zumindest im Sinne einer Absichtserklärung des Gesetzgebers - eine wichtige Rolle. Erst in der Phase der Umsetzung wird sich zeigen, ob das Gesetz $\mathrm{zu}$ realen Veränderungen hinsichtlich der Aus- und Weiterbildungssituation für Frauen führen wird.

\section{Geschlechterverhältnisse als Hintergrund}

Ein Blick auf die in der Schweiz herrschenden Geschlechterverhältnisse zeigt folgende, für den hier interessierenden Zusammenhang, wichtige Aspekte:

- Der Arbeitsmarkt ist in der Schweiz besonders stark geschlechtshierarchisch segregiert (Charles und Buchmann, 1994; Charles, 1995).

- Der segregierten Erwerbsarbeitswelt entsprechen die Lebensformen, die sich zwar auch in der Schweiz verändert und vervielfältigt haben, die sich aber nach wie vor - reell und normativ - stark am traditionellen Familienmodell (Ernährer/Hausfrau) orientieren (Eidgenössische Kommission für Frauenfragen, 1987). 
- Demgemäss sind die Möglichkeiten zur Vereinbarung von Beruf und Familie beschränkt und die Verantwortung für Haushalt und Kinderbetreuung lastet einseitig auf den Frauen (Borkowsky, Ley und Streckeisen, 1985 ).

Wenn wir die Bildungsentwicklung der letzten Jahrzehnte in der Schweiz betrachten, fällt sofort auf, dass die Frauen hinsichtlich Bildungsstand massiv aufgeholt haben. Formale Schranken, die Frauen von Ausbildungsgängen und dem Erwerb von Bildungsabschlüssen ausschlossen, sind praktisch vollständig gefallen. Doch stossen die Bildungsbemühungen von Frauen - sehr generell gesprochen - offensichtlich an Grenzen, die auf den eben genannten Aspekten basieren (Bundesamt für Statistik [BFS], 1996; Erziehungsdirektorenkonferenz [EDK], 1992; Mantovani Vögeli, 1994).

Die drei Aspekte sind eng miteinander verwoben, bedingen und stützen sich gegenseitig. Sie finden ihren Niederschlag sowohl in Infrastruktur, Recht, Sozialversicherung usw. wie auch in Betriebs- bzw. Berufskulturen und in den Einstellungen von Männern und Frauen. Und sie beeinflussen ganz massiv die Ausund Weiterbildungsmöglichkeiten von Frauen. Die Chancen wie auch die Benachteiligungen und Hindernisse, denen Frauen auf ihrem Bildungsweg begegnen, können nicht isoliert von diesem Kontext angegangen werden. Im erwähnten Bericht wird denn auch für eine umfassende Sichtweise plädiert.

Viel wurde von engagierten Frauen und Frauenorganisationen und seit 1981 (seit auch die Schweizer Verfassung einen Gleichstellungsartikel enthält) von den Gleichstellungsbeauftragten getan, um die Benachteiligung von Frauen in der beruflichen Aus- und Weiterbildung zu beheben. Ich meine aber, dass dabei das Problem lange zu einseitig (als Defizit) auf seiten der Frauen wahrgenommen und auch entsprechend bearbeitet wurde. Wirksame Problemlösungen und Chancenerweiterungen können aber nicht allein auf Bewusstseinsveränderung und Motivation von Frauen abzielen. Ebenso wichtig ist die Bearbeitung und Beseitigung der strukturell wirksamen Hindernisse.

Das Problem der beruflichen Diskriminierung von Frauen ist kein einfaches. Es gibt keine linearen Ursache-Wirkungs-Zusammenhänge. Die gegenseitigen Beeinflussungen von Strukturen, Kulturen, Einstellungen und Handlungsweisen sind komplex. Der notwendige Veränderungsprozess braucht viel Zeit und muss an verschiedensten Stellen ansetzen. Im Folgenden werden einige Ansatzpunkte aufgezeigt.

\section{Ansatzpunkte für die berufliche Gleichstellung von Frauen}

\section{Geschlechterstereotype Kompetenzförderung}

Im Rahmen eines internationalen Leistungsvergleichs von Schülerinnen und Schülern in Mathematik und Naturwissenschaften (Third International Mathe- 
mitics and Sience Study [TIMSS]) ging eine Forscherin der Frage nach, weshalb Mädchen in der Schweiz schlechtere Mathematikleistungen erbringen als Knaben. Die Ergebnisse machen deutlich, dass die Lehrpersonen, wie auch die Schülerinnen und Schüler, Mathematik als «männliches» Fach stereotypisieren. Entsprechend erwarten die Lehrerinnen und Lehrer weniger von den Mädchen und fördern sie auch weniger. Die Mädchen selbst entwickeln weniger Interesse, Leistungsbereitschaft und Selbstvertrauen in Bezug auf ihre Mathematikleistungen (Keller, 1998). Wichtig ist vor allem der letzte Punkt, das geringe Selbstvertrauen. Es führt dazu, dass Mädchen ihre reale Leistungsfähigkeit unterschätzen und Ausbildungen bzw. Studienrichtungen tendenziell meiden, in denen sie Kompetenzanforderungen vermuten, denen sie sich nicht gewachsen glauben.

Ähnliches spielt sich im Wissensbereich Physik ab. Mädchen zeigen auch im Fach Physik weniger Interesse, Begeisterung und Leistungsselbstvertrauen und sie wählen das Fach häufig ab, sofern ihnen Wahlfreiheit gewährt ist. Forschungsergebnisse zu dieser Problematik zeigen, dass die vorherrschenden didaktischen Ansätze im Physikunterricht den Mädchen zu wenig gerecht werden (Herzog et al., 1997).

Noch stärker männlich stereotypisiert ist m. E. der Bereich Technik. Lernbiografische Untersuchungen bei Ingenieurinnen und Frauen in Männerberufen weisen nach, dass viele von diesen Frauen über ihre Väter einen engen und persönlichen Bezug zu technischen Fragen gewannen und in ihrer Interessenentwicklung unterstützt und gefördert wurden. $\mathrm{Zu}$ vermuten ist, dass üblicherweise ein Widerspruch zwischen «Frau sein» und «sich für Technik interessieren» aufgebaut wird, der bei den oben erwähnten Frauen nicht zum Tragen kam (Blancpain, Bürgisser, Farago und Hüttner, 1988; Guhl, Rütter-Fischberger und Gasser, 1991; Sidler und Wettstein, 1982).

Bestrebungen zum Abbau von Stereotypisierungen ganzer Wissens-, Fachund Berufsbereiche richten sich an Eltern, Lehrpersonen sowie Schülerinnen und Schüler. Informationskampagnen, Spiele, Handbücher schon für den Kindergarten und die Unterstufe (Gehring und Marbot, 1997), Kursangebote in der Aus- und Weiterbildung von Lehrpersonen und didaktische Handreichungen (Labudde, 1999) zielen darauf ab, die Sensibilität gegenüber Stereotypisierungen zu erhöhen und den Akteurinnen und Akteuren Möglichkeiten aufzuzeigen für eine Erziehung und Unterrichtsgestaltung, die beiden Geschlechtern gerecht wird (Herzog et al., 1997).

"Geschlechterkulturen» in den Berufen

Technisch-handwerkliche Berufe haben oft ein «männliches» Image. Kraft und Umgang mit «hartem» Material werden betont, die zwischenmenschlichen Kontakte und Beziehungsaspekte (Zusammenarbeit, Kundenkontakt) werden verdrängt. Dienstleistungsberufe pflegen dagegen oft ein «weibliches» Image. Beziehungsaspekte werden betont, die erforderliche Kraft, Stressresistenz und Materialkenntnis werden in den Hintergrund gedrängt. 
Diese «Kulturen» werden immer wieder reproduziert und nach aussen auch dargestellt (Kantonales Amt für Berufsberatung Bern, 1988). Berufsbilder und Berufsangehörige transportieren sie und Stereotypen verstärken sie. Was heisst "Stereotypen» in diesem Zusammenhang? Die Berufswelt spielt sich heute vorwiegend hinter geschlossenen Türen ab. Unbefugte und insbesondere Kinder haben keinen Zutritt. Selten haben Kinder Gelegenheit, Berufsleute bei der Arbeit zu beobachten. Das heisst, ihre Vorstellungen von Berufen stützen sich auf wenige Informationen (Bétrisey, 1982; Frischknecht-Francfort, 1987). Nur das Offensichtlichste dringt ins Bewusstsein, das weniger direkt Wahrnehmbare bleibt im Verborgenen. Eine solche Offensichtlichkeit ist die zahlenmässige Übervertretung von Männern in technisch-handwerklichen Berufen und jene von Frauen im Dienstleistungsbereich. Damit ist beispielsweise das Stereotyp «für Dienstleistungsberufe, insbesondere im Erziehungs- und Pflegebereich, eignen sich Frauen besonders» bereits geboren. Und es ist hartnäckig; ebenso hartnäckig wie das Gegenstück, dass Frauen sich für die Arbeit mit «harten» Materialien und Maschinen nicht besonders eignen würden. Diese "Geschlechterkulturen» machen Frauen (und Männer), die in sogenannt untypischen Berufsdomänen arbeiten, immer «ungleich unter Gleichen», wie Heintz, Nadai, Fischer und Ummel (1997) nachweisen. Dabei wirkt sich das Ungleichsein unter Gleichen für Frauen negativer aus als für Männer, das heisst Frauen in Männerberufen haben mehr Akzeptanz-Probleme als Männer in der vergleichbaren Situation. Entsprechend dürfte es für eine junge Frau schwieriger sein, in einem Männerberuf Fuss zu fassen.

In aufwändigen Prozessen werden nun Berufsbilder und Unterlagen für die Berufsberatung umgestaltet, so dass sie möglichst beide Geschlechter ansprechen. Diese Gestaltungsarbeit bedeutet oft eine Gratwanderung zwischen einer stereotypenfreien oder "geschlechtsneutralen» und einer realistischen Darstellung der Berufe und Berufsfelder (Staufer und Christen, 1997). Auch «Tage der offenen Tür» können, sorgfältige und bewusste Planung vorausgesetzt, zur Relativierung von einseitigen Vorstellungen über Berufe beitragen. Auf recht intensives Interesse stossen die von technisch orientierten Ausbildungsstätten (z.B. Eidgenössische Technische Hochschule ETH in Zürich oder ABB Bildungszentrum in Baden) durchgeführten «Mädchen-Techniktage», während denen exklusive für Mädchen Ausbildungsmöglichkeiten im Bereich technischer Berufe vorgestellt werden. Diese Mädchen-Techniktage folgen der Zielsetzung, Vorbehalte, Ängste und Unsicherheiten seitens der Berufswählerinnen abzubauen, setzen ein gewisses Interesse der jungen Frauen an technischen Berufen aber voraus.

\section{Angst vor der Exponierung in einem "geschlechts- untypischen" Beruf}

Das zuvor erwähnte «Ungleichsein unter Gleichen» ist nicht unbedingt angenehm. Junge Frauen im Berufswahlalter schrecken davor zurück, als einzige Frau im Lehrbetrieb oder der Berufswahlklasse auftreten zu müssen. Dieses Gefühl 
des "Ausgestelltseins» hat verschiedene Aspekte. Oft stehen dahinter reale Erfahrungen von sexueller Belästigung und «Anmache» (Bähler, 1997). Sich dagegen täglich wehren zu müssen und sich wenn möglich auch noch den Vorwurf der "Zickigkeit» einzuhandeln, kann sehr belastend sein. Und längst nicht jeder Ausbildner, Personalchef oder Lehrmeister ist genügend sensibilisiert, um die Auszubildende vor entsprechenden Übergriffen im Betrieb schützen zu können (Arbogast und Seidenspinner, 1992). Ein weiterer Aspekt dieser Problematik ist der dauernde Beweiszwang. Der Beweis bezüglich Kompetenz muss von Frauen in «untypischen» Berufen immer wieder gegen die fragenden, ratlosen, zweifelnden Blicke oder gar entsprechende Bemerkungen erbracht werden, und auch das ist belastend. Ihren Anfang nehmen die Probleme aber schon im Augenblick, da ein Mädchen sich für einen "untypischen» Beruf zu interessieren beginnt, dieses Interesse mit Freundinnen und Freunden diskutiert und damit auf Unverständnis stösst. Möglicherweise fällt dieser Aspekt gar am stärksten ins Gewicht, denn allfällige Zweifel oder Ablehnung seitens der Gleichaltrigen greifen Jugendliche in einer biografischen Phase an, in der das Urteil der Gleichaltrigen von zentraler Bedeutung ist.

Diese Feststellungen legen nahe, dass junge Frauen auf dem Weg in einen «untypischen» Beruf begleitet werden müssen. Schlüsselpersonen wie betriebliche Ausbildnerinnen und Ausbildner, Berufsinspektorinnen und -inspektoren wie auch die Lehrpersonen an Berufsschulen müssen entsprechend sensibilisiert werden. Materialien dazu stehen seit Jahren zur Verfügung (z. B. Arbogast und Seidenspinner, 1992), doch ist deren Einsatz und Umsetzung keineswegs selbstverständlich, wie Galli (1990) am Fallbeispiel Tessin gezeigt hat. Mehr Effektivität verspricht der Einsatz von Frauen- oder Gleichstellungsbeauftragten an Ausbildungsstätten, wie er an eidgenössischen und kantonalen Hochschulen bereits üblich und nun auch für den Bereich der Fachhochschulen vorgesehen ist (NZZ, 10.11.1999, S. 15).

Neben den bis hierher erwähnten, eher indirekt wirksamen, häufig über einseitige Vorstellungen und Vorurteile transportierten Barrieren sind auch Hindernisse unmittelbar struktureller Art zu nennen.

\section{Strukturaspekte im Arbeitsalltag}

Gutes Betriebsklima, Kontakte zu Arbeitskolleg/innen sowie die Vereinbarkeit von Berufs- und Betreuungsarbeit sind wichtige Entscheidungskriterien in der Berufswahl junger Frauen (Schwarz-Türler, 1986). Im Prozess der Berufsfindung orientieren sich junge Frauen und Männer noch immer stark an traditionellen, für Frauen und Männer unterschiedlichen Laufbahnmustern (Grünewald-Huber, 1997). Beruf und Familie unter einen Hut bringen zu müssen, das ist eine Zumutung, mit der sich junge Frauen - und nur sie - in der Berufswahlphase konfrontiert sehen (Häfeli, 1983; Lemmermöhle, 1997). Und diese schwierige Leistung vollbringen zu können, erachten sie in Frauenberufen als leichter denn anderswo. Denn dort bringen ja offensichtlich viele Frauen die bei- 
den Arbeitsbereiche unter einen Hut. Zu welchem Preis ist wieder eine andere Frage, die oft erst dann schmerzhaft bewusst wird, wenn die Weichen längst gestellt sind (Ley, 1987).

Die Lösung des Vereinbarkeitsproblems steht mittlerweile auf der Agenda der Gleichstellungspolitik ganz oben. Für unseren Themenbereich wird es vor allem im Unterricht, der die Berufswahl vorbereitet und begleitet, relevant (Grossenbacher, 1994). Junge Frauen und junge Männer müssen in dieser Phase die Gelegenheit haben, sich mit Fragen der Vereinbarkeit von Beruf und Familie, mit Fragen der Rollenteilung und mit diesbezüglichen (traditionell orientierten) Erwartungen auseinandersetzen zu können. In dieser Perspektive wurden Materialien entwickelt (Zug um Zug, 1996; Von Windeln und Windows, o.J.), die sowohl für die individuelle Beschäftigung wie auch im Unterricht eingesetzt werden können.

\section{Unterschiedliche Ausbildungsdauer}

Solange Kurzlehren möglich sind, werden sie auch gewählt. Allerdings werden sie vor allem im Bereich der von Frauen dominierten Berufe angeboten und entsprechend viel häufiger von jungen Frauen als von jungen Männern gewählt. Junge Frauen finden in diese Kurzlehren, weil sie sich für die Arbeitsinhalte der entsprechenden Berufe interessieren oder weil sie so rasch als möglich ins Erwerbsleben einsteigen und finanziell unabhängig sein wollen oder weil die Eltern gegen eine grössere Bildungsinvestition sind. Absolventinnen von Kurzlehren stellen keine verschwindende Minderheit dar, wie man gerne glauben möchte. Anfangs der 90er Jahre waren 25\% der weiblichen Auszubildenden in einer Lehre, die bis zu zwei Jahren dauerte. Auch wenn für die Ausübung der entsprechenden Berufe eine Lehrdauer von zwei Jahren genügt, bedeuten Kurzlehren für die Absolventinnen, dass sie weniger berufliche Kenntnisse und vor allem weniger Allgemeinbildung vermittelt bekommen als Lehrlinge in drei- und vierjährigen Lehren (Borkowsky und Gonon, 1996, S. 27). Sie werden zudem beim Zugang zu formalen Weiterbildungsmöglichkeiten diskriminiert, weil dort oft drei- oder vierjährige Lehren vorausgesetzt werden. Als Beispiel sei die Berufsmatur genannt, die nur im Zusammenhang oder nach Abschluss einer mindestens dreijährigen Lehre gemacht werden kann.

Selbstverständlich kann man versuchen, junge Frauen von Kurzlehren «wegzuberaten». Sinnvoller und wirksamer aber ist eine Angleichung der Lehrdauern, wie sie im Entwurf zum neuen Berufsbildungsgesetz vorgesehen ist. Für bereits Berufstätige sind Weiterbildungsmöglichkeiten wichtig, die im Sinne der Nachholbildung den Erwerb von Grundqualifikationen auch im Erwachsenenalter und die Ergänzung von kurzen Erstausbildungen ermöglichen (Schräder-Naef, 1997). 


\section{Strukturaspekte im Ausbildungs- und \\ Weiterbildungsbereich}

Weitere Stolpersteine der strukturellen Art kennzeichnen den Ausbildungs- und Weiterbildungsbereich. Die zeitliche Organisation oder die geografische Entfernung von Ausbildungs- und Weiterbildungsmöglichkeiten können für Frauen mit Familienpflichten als Barriere wirken. So schön und erwachsenenbildnerisch hochwirksam Blockkurswochen im Grünen sind, so stellen sie doch z.B. alleinerziehende Mütter vor fast unlösbare Organisationsprobleme. Das gleiche gilt für Vorgaben, die im Bereich der berufsbegleitenden Ausbildungen eine hohe zeitliche Belastung durch Berufsarbeit und Aus-/Weiterbildung bewirken. Wenn neben einer berufsbegleitenden Ausbildung 60-80\% Berufstätigkeit verlangt werden, dann wirkt das für Personen mit Familienpflichten prohibitiv. In diesem Sinne hinderlich sind auch «Hausaufgaben». Untersuchungen zum Lehrabschluss nach Artikel 41 Berufsbildungsgesetz haben gezeigt, dass Frauen weniger auf die Abschottung gegen familienbedingte Störungen zählen können und dass sie auch weniger Gelegenheit haben, im Geschäft ihre Aufgaben zu machen (Häfeli, 1991). Diskriminierend wirken schliesslich Altersgrenzen bei Aus- und Weiterbildungsangeboten, ebenso wie die enge Bezogenheit von Weiterbildungsangeboten auf einen Ausbildungsberuf.

Positiv zu werten sind die Bemühungen um Flexibilisierung von Weiterbildungsmöglichkeiten. Weiterbildung, die im Baukastensystem angeboten wird, ist ein wichtiger Beitrag zum Abbau struktureller Barrieren (Projektleitung «Berufliche Weiterbildung im Baukastensystem», 1999), sofern gleichzeitig ein allgemein anerkannter Nachweis der erworbenen Qualifikationen möglich ist, in den auch die ausserberuflich erworbenen Qualifikationen mit einbezogen sind. Letzteres ist vor allem für Wiedereinsteigerinnen von zentraler Bedeutung (Sautebin-Pousse, 1995).

\section{Vorurteile von Vorgesetzten}

Personalverantwortliche und Ausbildende fördern Frauen weniger, wenn sie Vorurteile haben (z.B. bezüglich Leistungsfähigkeit, Weiterbildungsinteresse, Aufstiegswille, Betriebstreue, Flexibilität etc.). Wenn eine ausbildende Person der Meinung ist, Frauen seien prinzipiell weniger leistungsfähig, dann wird sie den Unterricht für Frauen weniger herausfordernd gestalten und wird damit den gleichen fatalen Mechanismus in Gang setzen, der eingangs im Zusammenhang mit dem Fach Mathematik schon erwähnt wurde. Oft braucht es Vorgaben von der höchsten Führungsebene oder Quotenregelungen, damit Personalverantwortliche auch Frauen für die Weiterbildung und den Aufstieg vorschlagen. Und manchmal braucht es Vorgaben und Quotenregelungen und den Vorschlag des Vorgesetzten, damit Frauen sich den nächsten Schritt vorwärts und aufwärts zutrauen (Mussmann, 1990; Nadai, 1992).

Um die Wirksamkeit von Weiterbildungsangeboten für Frauen und insbesondere von Angeboten, die spezifisch für Frauen ausgearbeitet und nur für 
Frauen angeboten wurden zu prüfen, wurden solche Angebote im Rahmen der öffentlichen Verwaltung evaluiert. Sie erwiesen sich vor allem dann als wirkungsvoll, wenn sie von den Vorgesetzten befürwortet und der Transfer entsprechend unterstützt wurde (Stalder, 1995).

\section{Mangelnde Laufbahnplanung}

Für Frauen scheint es weniger selbstverständlich zu sein, ihre berufliche Laufbahn zu planen und die Weiterentwicklung gezielt voranzutreiben. Exemplarisch zeigt das eine Studie von Lisbeth Hurni und Barbara Stalder, die unter dem sprechenden Titel «Ich verplane mir das Leben nicht gern auf Jahre ...» vom Eidg. Büro für die Gleichstellung von Frau und Mann herausgegeben wurde (Hurni und Stalder, 1994). Mangelnde Laufbahnplanung ist aber nicht nur eine «Unterlassungssünde» der Frauen selbst. Sie wird auch im Mitarbeiterinnen-Gespräch, in der beruflichen Beratung und während der Ausbildung zu wenig thematisiert (Mantovani Vögeli und Grossenbacher, 1988; Meili-Lüthi, 1995). Während der Ausbildung lassen sich sogar Prozesse beobachten, die Frauen und Männer in gleichen Ausbildungsberufen in unterschiedliche Laufbahnmuster "hineinqualifizieren». So werden weibliche Auszubildende im kaufmännischen Bereich in Richtung Sekretariat "gefördert», ihre Kollegen hingegen in Richtung Sachbearbeitung und Rechnungswesen (Diemand und Lohner, 1985; Heintz et al., 1997).

Mit der zunehmenden Bedeutung, die Bildungsabschlüssen, Qualifikationszertifikaten und lebenslangen Bildungsaktivitäten zukommt, gewinnt auch die Laufbahnplanung an Wichtigkeit. Beratungs- und Unterstützungsangebote sind auch im Zusammenhang mit der Modularisierung im Weiterbildungssystem notwendig, denn diese Entwicklungen machen den Weiterbildungsbereich - zumindest in einer mittleren Zeitperspektive - nicht transparenter. Unabdingbar werden solche Angebote für Menschen, die sich im Aus- und Weiterbildungsbereich, der zunehmend marktförmig organisiert wird, mit mehrfachen Diskriminierungen konfrontiert sehen.

\section{Kumulative Diskriminierung}

Ursache für berufliche Diskriminierung ist nicht nur die Geschlechtszugehörigkeit. Mangelnde Kenntnisse der vorherrschenden Sprache, Zugehörigkeit zu einer kulturellen Minderheit, Alter, Krankheit, Behinderung sowie Belastungen durch Familienpflichten sind weitere Faktoren, die zu Diskriminierungen Anlass geben können. Und nur zu oft wirken die Diskriminierungen kumulativ. Für Ausländerinnen, die der vorherrschenden Sprache zu wenig mächtig sind, bestehen kaum zugängliche Bildungsangebote, wie Luciana Rovis und Mitarbeiterinnen aufgezeigt haben (Rovis, Loppacher-Heizmann und Steinmann, 1995). Auch Bildungsangebote, die von der Idee her sehr geeignet wären, wie der Lehrabschluss für Erwachsene (nach Art. 41 des Berufsbildungsgesetzes), sind den Bedürfnissen dieser Frauen nicht angepasst. Doch die Probleme beginnen auch 
hier schon im Vorfeld der Berufswahl, wo ausländische Mädchen ihre Situation zwischen den Kulturen häufig als unüberwindliches Hindernis empfinden (Kraft, 1980; Scandroglio, 1985).

Diese kumulativen Diskriminierungen erscheinen - aus der Logik des hier Vorgetragenen heraus - erst am Schluss. Und bei der Konzipierung von Bildungsmassnahmen gehen sie oft ganz vergessen (Marti, 1994). Von der Problemrelevanz her müssen sie jedoch am Anfang stehen. Denn es sind diese kumulativen Diskriminierungen, an denen sich die Bemühungen um Gleichstellung letztlich messen lassen müssen. Als Fazit für die 80er Jahre musste, trotz bereits fast 10jähriger Gültigkeit des Gleichstellungsartikels in der Verfassung, festgehalten werden, dass berufliche Gleichstellung noch immer ein Mythos sei (Charles, 1995). In den neunziger Jahren wurde das Gleichstellungsgesetz erlassen, das die Möglichkeit gewährt, Massnahmen zur Förderung der Gleichstellung finanziell zu unterstützen. In der Folge sind zahlreiche Projekte in diesem Rahmen ausgearbeitet und gestartet worden. Gleiches gilt für die Weiterbildungsoffensive und die Lehrstellenbeschlüsse, die ebenfalls Kampagnen und Projekte mit der Zielperspektive «Förderung der Gleichstellung» ermöglichten.

Zum Schluss muss festgehalten werden, dass Gleichstellung nicht primär ein Bildungsproblem ist, auch wenn gleicher Zugang zu Bildung eine wichtige Voraussetzung für Gleichstellung darstellt und Gleichstellung selbstverständlich auch auf allen Ebenen des Bildungsbereiches gewährleistet sein muss. Wie dem Postulat "Gleichstellung im Bildungsbereich» nachgelebt werden kann, wurde in den vorhergehenden Ausführungen ansatzweise aufgezeigt. Daneben braucht es aber grundsätzlichere gesellschaftliche Veränderungen, die auf die eingangs erwähnten Geschlechterverhältnisse im Hintergrund abzielen. Solange die Verdienstmöglichkeiten in frauendominierten Berufen nicht existenzsichernd sind und diese Berufe keine Entwicklungsperspektiven bieten, und solange die Beschäftigungsbedingungen im männerdominierten Bereich nicht flexibler werden, wird sich weder an der geschlechtshierarchischen Segregation am Arbeitsmarkt etwas ändern, noch wird die Vereinbarkeit von Berufs- und Familienarbeit erleichtert. Und umgekehrt - solange die Vereinbarkeit von Beruf und Familie nicht durch ein befriedigendes Angebot an Kinderbetreuungsmöglichkeiten und durch eine adäquate Absicherung in der Sozialversicherung für Männer und Frauen gewährleistet ist, werden Männer weiterhin Ernährerlöhne verdienen und Frauen beruflich zugunsten der Familie zurückstecken müssen. In diesem Sinne geht es auf dem Weg zur Gleichstellung weniger um «Frauenförderung» als vielmehr um die Schaffung gesellschaftlicher Rahmenbedingungen, die eine wirklich freie Wahl sowohl der Lebensform wie auch des Berufes für beide Geschlechter ermöglichen und eine partnerschaftliche Ausgestaltung des Alltags zwischen Beruf und Familie zulassen. 


\section{Literatur}

Arbogast, C. \& Seidenspinner, G. (1992). Mädchen ausbilden im gewerblich-technischen Bereich. Weinheim \& Basel: Beltz.

Bähler, R. (1997). Frauenlaufbahnen in Männerdomänen. Panorama, (6), 24-26.

Bétrisey, G. (1982). Les intérêts des garçons et des filles de 13 à 15 ans. Orientation et formation professionnelles, 1, 41-45.

Blancpain, R., Bürgisser, M., Farago, P. \& Hüttner, E. (1988). Frauen im Ingenieurberuf. IPSO-Studie im Auftrag der Akademie für technische Wissenschaften SATW. Zürich: SATW.

Borkowsky, A., Ley, K. \& Streckeisen, U. (1985). Strukturelle und subjektive Aspekte von Arbeitsbiografien, Erwerbsverläufen und Berufslaufbahnen von Frauen. Schlussbericht. Bern: Nationalfond.

Borkowsky, A. \& Gonon, Ph. (1996). Berufsbildung in der Schweiz. Beteiligung gestern und heute - neue Herausforderungen. Bern: Bundesamt für Statistik.

Bundesamt für Statistik BFS (1996). Auf dem Weg zur Gleichstellung. Frauen und Männer in der Schweiz. Zweiter statistischer Bericht. Bern: BFS.

Bundesamt für Berufsbildung und Technologie BBT (Hrsg.) (1998). Berufliche Aus- und Weiterbildung von Frauen. Bericht zum Postulat von Nationalrätin Ruth Grossenbacher. Bern: BBT.

Burri, R., Fleischmann, I. \& Pagnossin-Aligisakis, E. (1998). Gender Studies /Women's Studies in der Schweiz. Bestandesaufnahme Lehre und Forschung 1995-1997. Bern: Schweizerischer Wissenschaftsrat (FOP 51/1998d).

Charles, M. (1995). Berufliche Gleichstellung - ein Mythos. Hrsg. vom Bundesamt für Statistik. Bern: BFS.

Charles, M. \& Buchmann, M. (1994). Assessing micro-level explanations of occupational sex segregation: human-capital development and labor market opportunities in Switzerland. Schweizerische Zeitschrift für Soziologie, (3), 595-620.

Diemand, Ch. \& Lohner, D. (1985). Geschlechtsspezifische Unterschiede in der nicht-fachlichen Ausbildung von Lehrtöchtern und Lehrlingen. Zürich: Universität, Psychologisches Institut (unveröffentlichte Lizentiatsarbeit).

Eidgenössische Kommission für Frauenfragen (1987). Frauen und Männer: Fakten, Perspektiven, Utopien. Bern: EKF.

Erziehungsdirektorenkonferenz EDK (1992). Mädchen - Frauen - Bildung. Unterwegs zur Gleichstellung. Bern: EDK.

Frischknecht-Francfort, J. (1987). Les projets professionnels des adolescentes. In S. LempenRicci \& Th. Moreau (Ed.), Vers une éducation non sexiste (S. 109-121). Lausanne: Edition Réalités sociales.

Galli, E. (1990). Formazione della giovane: possibilità e limiti nel Cantone Ticino. Bellinzona: DPE, USR e SFP.

Gehring, G. \& Marbot, M. (1997). Wir lassen Rollen rollen: Thema Berufs- und Geschlechterrollen in Kindergarten und Unterstufe. Bern \& Gümligen: Zytglogge.

Grossenbacher, S. (1994). Berufs- und Schulwahlvorbereitung: Fächerübergreifende Gestaltung des berufs- und schulwahlvorbereitenden Unterrichts an Schulen der Sekundarstufe I. Liestal: Erziehungs- und Kulturdirektion Basel-Landschaft; Büro für die Gleichstellung von Frau und Mann.

Grünewald-Huber, E. (1997). Koedukation und Gleichstellung. Eine Untersuchung zum Verbältnis der Geschlechter in der Schule. Chur \& Zürich: Rüegger.

Guhl, D., Rütter-Fischberger, U. \& Gasser, U. (1991). Frauen in technischen Berufen: Ergebnisse einer Befragung zur beruflichen Situation von IngenieurInnen, ArchitektInnen und InformatikerInnen. Zürich: INGCH; SIA.

Häfeli, K. (1983). Die Berufsfindung von Mädchen: zwischen Familie und Beruf. Bern: Lang.

Häfeli, K. (1991). Die Lehrabschlussprïfung für Erwachsene. Zürich: Institut für Bildungsforschung und Berufspädagogik im Amt für Berufsbildung des Kantons Zürich (Berichte Band 23). 
Heintz, B., Nadai, E., Fischer, R. \& Ummel, F. (1997). Ungleich unter Gleichen. Studien zur geschlechtsspezifischen Segregation des Arbeitsmarktes. Frankfurt a. Main: Campus.

Herzog, W., Labudde, P., Neuenschwander, M.P., Violi, E. \& Gerber, C. (1997): Koedukation im Physikunterricht. Schlussbericht zuhanden des Schweizerischen Nationalfonds zur Förderung der wissenschaftlichen Forschung. Bern: Universität Bern.

Hurni, L. \& Stalder, B. (1994). "Ich verplane mir das Leben nicht gern auf Jahre ...». Berufliche Entwicklungsmuster bei jungen Frauen und Männern. Pilotstudie über Entscheidungstendenzen und Wunschvorstellungen zu Familie und Beruf. Bern: Eidg. Büro für die Gleichstellung von Frau und Mann.

Kantonales Amt für Berufsberatung Bern (1988). Frauen und Männer in allen Berufen - wie informiert die Berufsberatung zu Berufsmann und Berufsfrau? Bern: Kantonales Amt für Berufsberatung.

Keller, C. (1998). Geschlechterdifferenzen in der Mathematik: Prüfung von Erklärungsansätzen. Eine mehrebenen-analytische Untersuchung im Rahmen der "Third International Mathematics and Science Study». Zürich: Universität (Dissertation).

Kraft, U. (1980). Auswirkungen der Migration auf Berufsfindungsprozesse jugendlicher Ausländer. Zürich: Universität (unveröffentlichte Lizentiatsarbeit).

Labudde, P. (1999). Mädchen und Jungen auf dem Weg zur Physik. Naturwissenschaften im Unterricht Physik, (10), 4-10.

Lemmermöhle, D. (1997). Geschlechterverhältnisse und Bildung - Überlegungen zu einer veränderten Mädchen- und Jungenbildung. In S. Grossenbacher, W. Herzog, F. Hochstrasser, R. Rüegsegger (Hrsg.), Schule und soziale Arbeit in gefährdeter Gesellschaft (S. 259275). Bern: Haupt.

Ley, K. (1987). Ich hätte lieber etwas anderes gelernt.... In Schweizerischer Verband für Berufsberatung (Hrsg.), Zwischen Qual und Qualifikation. Band I: Berufswahl. Zürich: Schweizerischer Verband für Berufsberatung.

Mantovani Vögeli, L. (1994). Fremdbestimmt zur Eigenständigkeit. Mädchenbildung gestern und heute. Chur \& Zürich: Rüegger.

Mantovani Vögeli, L. \& Grossenbacher, S. (1988). Frauenförderungsmassnabmen für angehende kaufmännische Angestellte. Vorprojekt des Bundes Schweizerischer Frauenorganisationen. Bern: Bund Schweizerischer Frauenorganisationen.

Marti, C. (1994). Weiterbildung von Ausländern und neue Technologien (WANT): Schlussbericht. Zürich: ETHZ, Institut für Arbeitspsychologie.

Meili-Lüthi, E. (1995). Laufbahnplanung bei jungen Frauen nach der Erstausbildung: Implikationen für die Berufsberatung. Schwerzenbach: Eigendruck.

Mussmann, C. (1990). Frauen im Verkauf: Chancen und Barrieren bei Weiterbildung und Aufstieg. Zürich: Kaufmännischer Verband Zürich (KVZ).

NZZ, 10.11.1999. Innovative Forschung, träge Strategien. Zwischenbilanz des Bundes zur Fachhochschulreform (S. 15).

Nadai, E. (1992). Akademische Laufbahn und Geschlecht. Bern: Bundesamt für Statistik

Projektleitung «Berufliche Weiterbildung im Baukastensystem»(Hrsg.) (1999). Berufliche Weiterbildung im Baukastensystem. Schlussbericht über die Pilotphase 1996 bis 1998. Erfahrungen, Schlussfolgerungen und Vorschläge der Projektleitung an das Bundesamt für Berufsbildung und Technologie (BBT). Bern: Bundesamt für Berufsbildung und Technologie (BBT).

Rovis, L., Loppacher-Heizmann E. \& Steinmann, B. (1995). Fremdsprachige Arbeitnehmerinnen und Berufsbildung. Basel: ECAP.

Sacchi, S. \& Buchmann, M. (1997). Determinanten und Effekte beruflicher Weiterbildung wichtige Befunde und Implikationen. Bulletin SGBF, (2), 33-36.

Sautebin-Pousse, M.-T. (1995). Femmes au foyer: une identité à l'épreuve. Genève: Université, FPSE (mémoire de licence non publié). 
Scandroglio, R. (1985). Aspekte der Sozialisations- und Berufswahlsituation von Italienermädchen im Jugendalter. Zürich: Universität, Pädagogisches Institut (unveröffentlichte Lizentiatsarbeit).

Schräder-Naef, R. (1997). Warum Erwachsene (nicht) lernen. Zum Lern- und Weiterbildungsverhalten Erwachsener in der Schweiz. Chur \& Zürich: Rüegger.

Schwarz-Türler, M. N. K. (1986). «...die Mädchen ermuntern, auch ungewohnte und anspruchsvolle Berufe zu wählen». Berufsberatung und Berufsbildung, (5), 273-283.

Schweizerischer Bundesrat (1996). Bericht über die Berufsbildung (Bundesgesetz über die Berufsbildung), vom 11. September 1996. Bern: EDMZ.

Sidler, R. \& Wettstein, E. (1982). Frauen in Männerberufen. Berufliche Situation von Frauen, die einen sogenannten Männerberuf erlernt haben. Zürich: Institut für Bildungsforschung und Berufspädagogik im Amt für Berufsbildung des Kantons Zürich.

Stalder, B. (1995). Frauenspezifische Weiterbildung im Betrieb. Zürich: Verlag der Fachvereine vdf.

Staufer, H. \& Christen, H. (1997). Leider keine Mauernden. Panorama, (6), 12-13.

Von Windeln und Windows (o.J.). Herausgegeben vom Büro für die Gleichstellung von Frau und Mann der Stadt Zürich. Heft 3: je eine Ausgabe für Mädchen, für Knaben, für Mütter/Väter. Zürich: Büro für die Gleichstellung von Frau und Mann.

Zug um Zug (1996). Mädchen und Jungen - auf zu einer offenen Berufswahl. (Helgard Christen und Ursula Huber). Zürich: sabe. 


\section{Les femmes dans la formation professionnelle initiale et continue. Conséquences pour la question du genre dans la formation professionelle.}

\section{Résumé}

L'article met en évidence les problèmes et discriminations avec lesquels beaucoup de femmes sont confrontées pendant leur carrière scolaire et professionnelle. Il fait référence aux differentes phases: orientation, formation de base, formation continue et réintégration professionnelle. Critiquant le fait que jusqu'à présent les mesures pour l'égalité entre hommes et femmes étaient étroitement orientées sur l'information et la motivation des femmes, l'auteur montre des approche plutôt orientées sur des changements structurels.

\section{Le donne nella formazione professionale di base e continua. Conseguenze per la questione dell'appartenenza sessuale}

Riassunto

Questa contribuzione espone i problemi e le discriminazioni che molte donne devono confrontare durante il loro iter scolastico e professionale. L'articolo fa riferimento alle diverse fasi di questo iter: orientamento, formazione di base, riqualificazione, reintegrazione nella vita professionale. L'autrice deplora il fatto che finora le misure tendenti ad aumentare l'ugualianza dei sessi si sono più o meno limitati all'informazione e alla motivazione delle donne; in complemento descrive alcune misure che vanno nella direzione di cambiamenti strutturali.

\section{Women in vocational training and continuing education. Consequences for gender issues in vocational training.}

\section{Summary}

The article examines problems and discriminations women are confronted with during their school and professional career and its different phases: orientiation, basic and further vocational training and re-integration. Criticizing that campains for eqality hitherto were based on information and motivation of women the author shows approches mainly based on structural change. 